\title{
Association of pretreatment serum carcinoembryonic antigen levels with chemoradiation-induced downstaging and downsizing of rectal cancer
}

\author{
SEUNG-GU YEO \\ Department of Radiation Oncology, Soonchunhyang University College of Medicine, \\ Soonchunhyang University Hospital, Cheonan 31151, Republic of Korea \\ Received January 29, 2015; Accepted January 13, 2016
}

DOI: $10.3892 / \operatorname{mco} .2016 .740$

\begin{abstract}
The aim of this study was to identify pretreatment clinical parameters associated with preoperative chemoradiotherapy (CRT)-induced downstaging and downsizing of locally advanced rectal cancer (LARC T3-4 or N+). Data from 51 LARC patients, who received preoperative CRT and radical surgery between 2010 and 2013, were retrospectively analyzed. Rectal adenocarcinoma was histologically confirmed in all patients, who ranged in age between 41 and 81 years (median, 64 years). CRT consisted of 50.4 Gy pelvic radiotherapy with concurrent chemotherapy using 5-fluorouracil and leucovorin. After a median interval of 7 weeks post-CRT, the patients underwent total mesorectal excision. Downstaging was defined as the transition from cStage II-III to ypStage 0-I. The longest tumor diameter was measured pre- and post-CRT using computed tomography or magnetic resonance imaging, and based on the surgical specimen, respectively. Downstaging was observed in $16(31.4 \%)$ patients, including $5(9.8 \%)$ with a pathological complete response. The median downsizing rate was $60 \%$. The serum carcinoembryonic antigen (CEA) levels were $0.8-153.9 \mathrm{ng} / \mathrm{ml}$ (median, $4.4 \mathrm{ng} / \mathrm{ml}$ ). The maximum standardized uptake value was 4.7-33.9 (median, 10.8). On univariate analysis, cT stage, tumor size and CEA level were associated with downstaging. On multivariate analysis, only CEA level $(\leq 5 \mathrm{ng} / \mathrm{ml})$ was a significant predictor of downstaging (odds ratio $=16.0 ; 95 \%$ confidence interval: $1.8-146.7$; $\mathrm{P}=0.014)$. CEA level was the only factor significantly associated with downsizing $(>60 \%)$ in the univariate analysis. These results demonstrated that pretreatment serum CEA levels are significantly associated with downstaging as well as downsizing of LARC following preoperative CRT. Therefore, this
\end{abstract}

Correspondence to: Dr Seung-Gu Yeo, Department of Radiation Oncology, Soonchunhyang University College of Medicine, Soonchunhyang University Hospital, 31 Soonchunhyang 6-gil, Cheonan 31151, Republic of Korea

E-mail: md6630@schmc.ac.kr

Key words: rectal cancer, carcinoembryonic antigen, chemoradiotherapy, downstaging, downsizing parameter may be useful in personalizing the management of LARC patients.

\section{Introduction}

The preferred standard treatment for locally advanced rectal cancer (LARC, T3-4 or N+) includes preoperative short-course radiotherapy (RT) or long-course chemoradiotherapy (CRT), followed by radical surgery and postoperative chemotherapy $(1,2)$. However, employing this multimodal treatment approach for all patients with LARC may constitute overtreatment for a subset of the patients. Both preoperative therapy or radical surgery may be considered overtreatment under certain circumstances (3). A more personalized approach to the management of LARC patients has been attracting increasing attention (4).

The response of LARC to CRT varies from absent to complete (5). To implement personalized treatment for LARC, early and accurate prediction (or assessment) of CRT response is necessary (6). The predicted response to CRT may affect preoperative treatment, while response assessment post-CRT may affect the surgical approach. Certain patients may derive no benefits from preoperative CRT or radical surgery, whereas they may suffer from treatment-induced morbidities.

The aim of this study was to investigate the pretreatment clinical, patient and tumor parameters regarding their association with LARC response to CRT, which was indexed by the downstaging and downsizing of rectal cancer.

\section{Patients and methods}

Patients. Data from 51 LARC patients who were managed with preoperative CRT and radical surgery between 2010 and 2013 at the Soonchunhyang University Hospital (Cheonan, Korea) were retrospectively analyzed. Rectal adenocarcinoma was pathologically proven in all the cases. The clinical stage was T3-4 or N+ (cStage II-III), evaluated using abdominopelvic computed tomography (CT), pelvic magnetic resonance imaging (MRI), and/or endorectal ultrasonography. The Institutional Ethics Committee approved the study protocol (2015-01-006), and waived the written informed consent from the patients due to the retrospective nature of the study. 
Pretreatment assessment. The pretreatment staging work-up included a digital rectal examination, complete blood count, liver function tests, assessment of serum carcinoembryonic antigen (CEA) levels, and colonoscopy with rectal biopsy. Chest radiography, abdominopelvic CT, pelvic MRI, ${ }^{18} \mathrm{~F}$-fluorodeoxyglucose positron emission tomography-CT, and/or endorectal ultrasonography were also performed.

For preoperative RT, the patients underwent CT simulation. The initial target volume encompassed the gross tumor, mesorectum, presacral space and regional lymphatics (perirectal, presacral, internal iliac and distal common iliac). A three-dimensional conformal plan comprised a 6-MV photon posterior-anterior field and 15-MV photon opposed lateral fields. RT at a dose of 45 Gy was delivered in 25 fractions to the pelvis, with a continuous boost of 5.4 Gy delivered in 3 fractions. The boost target volume involved the gross tumor and adjacent mesorectum. Preoperative chemotherapy, administered concurrently with RT, used two cycles of a bolus infusion of 5 -fluorouracil $\left(450 \mathrm{mg} / \mathrm{m}^{2} / \mathrm{d}\right)$ and leucovorin $\left(20 \mathrm{mg} / \mathrm{m}^{2} / \mathrm{d}\right.$ ) for 5 days during the first and last weeks of RT.

Tumor resection and post-treatment staging. Six to eight weeks after preoperative CRT completion, the patients underwent radical resection, including total mesorectal excision. A total of 45 patients underwent low anterior resection, 3 received Hartmann's operation, and the remaining 3 were subjected to abdominoperineal resection. The post-CRT pathological stage (ypStage) was determined according to the American Joint Committee on Cancer (AJCC) staging system, 7th edition (7). Downstaging was defined as initial cStage II-III changing to ypStage 0-I following CRT. Tumor size was indexed by the maximum size of the rectal mural tumor on pretreatment $\mathrm{CT}$ or MRI, and the post-CRT residual viable tumor on the surgical specimen. Downsizing was indicated by a tumor size reduction of $>60 \%$, which represented the median value in all patients.

Statistical analysis. To identify the pretreatment parameters associated with pathological response to CRT, the Chi-square or Fisher's exact test were used for the univariate analyses. Multivariate logistic regression was performed, including the factors that achieved statistical significance in the univariate analyses. The CRT response was indexed by downstaging as well as downsizing. All reported P-values were two-tailed and a P-value of $<0.05$ was considered to indicate statistically significant differences. All analyses were performed using the SPSS software, version 14.0 (SPSS Inc., Chicago, IL, USA).

\section{Results}

Patient characteristics. Of a total of 51 patients, ranging in age between 41-81 years (median, 64 years), 35 (68.6\%) were male. The cT classification was cT2 in $5(9.8 \%)$, cT3 in $32(62.7 \%)$, and cT4 in $14(27.5 \%)$ patients. The cStage was II in 10 (19.6\%) and III in $41(80.4 \%)$ patients. The pretreatment CEA levels and maximum standardized uptake value were specified in 45 and 44 patients, respectively, with values of $0.8-153.9 \mathrm{ng} / \mathrm{ml}$ (median, $4.4 \mathrm{ng} / \mathrm{ml}$ ) and 4.7-33.9 (median, 10.8), respectively.

Post-treatment classification. The post-CRT ypT classification was ypT0 in 2 (3.9\%), ypTis in 3 (5.9\%), ypT1 in $3(5.9 \%)$,
ypT2 in $12(23.5 \%)$, ypT3 in $28(54.9 \%)$ and ypT4 in $3(5.9 \%)$ patients. The ypStage was 0 in $5(9.8 \%)$, I in $11(21.6 \%)$, II in $19(37.3 \%)$, and III in $16(31.4 \%)$ patients. Downstaging was observed in $16(31.4 \%)$ patients. The pre- and post-CRT tumor sizes were $2-11 \mathrm{~cm}$ (median, $4.8 \mathrm{~cm}$ ) and $0-5 \mathrm{~cm}$ (median, $2 \mathrm{~cm}$ ), respectively. The median reduction in tumor size was $60 \%$ (range, $0-100 \%$ ). Downsizing (>60\%) was observed in 25 (49\%) patients.

Factors associated with downstaging and downsizing. The results of the univariate analyses of pretreatment factors associated with downstaging are presented in Table I. cT classification, tumor size and CEA level were found to be significantly associated with downstaging. On multivariate analysis, only CEA level $(\leq 5 \mathrm{ng} / \mathrm{ml})$ significantly predicted downstaging [odds ratio $(O R)=16.0 ; 95 \%$ confidence interval (CI): $1.8-146.7 ; \mathrm{P}=0.014]$. In the univariate analyses of factors associated with downsizing (Table II), only CEA level was found to be significant.

\section{Discussion}

The present study analyzed the pretreatment clinical, patient and tumor parameters to identify the factors associated with response to CRT, and the serum CEA levels were found to be significantly associated with CRT-induced LARC downstaging and downsizing.

Serum CEA is the most widely used tumor marker in colorectal cancer. In 2000, the AJCC stated that preoperative elevation of CEA ( $\geq 5 \mathrm{ng} / \mathrm{ml}$ ) merits inclusion as a category I prognostic factor in colorectal cancer (8). Since the timing of CRT has changed from postoperative to preoperative in LARC, ongoing research has sought to determine the markers associated with CRT response, which is ascertained early through surgical pathology. If the previously recognized prognostic value of preoperative CEA was related to the effectiveness of postoperative CRT, then CEA level may also be associated with preoperative CRT response.

Several previous studies have similarly reported that pre-CRT CEA level was associated with CRT response in rectal cancer (9-12). Park et al (9) analyzed data from 141 rectal cancer patients, categorizing their CRT response as good or poor. The good response group $(n=26 ; 19 \%)$ included patients with pathologically complete or near-complete responses. The mean pre-CRT level of CEA was $18.99 \mathrm{ng} / \mathrm{ml}$ in the poor response group and $6.57 \mathrm{ng} / \mathrm{ml}$ in the good response group $(\mathrm{P}=0.049)$. The multivariate analysis indicated that an elevated pre-CRT CEA level $(>5 \mathrm{ng} / \mathrm{ml})$ was the only significant predictor of a poor response $(\mathrm{OR}=3.030 ; 95 \% \mathrm{CI}$ : $1.03-8.26)$. Wallin et al (10) analyzed data from 469 rectal cancer patients and reported that the factors associated with a pathological complete response $(n=96 ; 20 \%)$ on multivariate analysis were low $(\leq 5 \mathrm{ng} / \mathrm{ml})$ pretreatment CEA levels $(\mathrm{OR}=0.87$; 95\% CI: 0.77-0.97) and interruption of CRT. Pretreatment cT classification and tumor size were significantly associated with T-stage down-classification, but no parameters were associated with a reduction in tumor size. In the present study, statistically significant factors associated with pathological complete response were not identified, possibly due to the small patient sample. Instead, it was observed that pretreatment CEA levels 
Table I. Univariate analysis of factors associated with downstaging of locally advanced rectal cancer.

\begin{tabular}{|c|c|c|c|c|c|}
\hline \multirow[b]{2}{*}{ Factors } & \multicolumn{2}{|c|}{ ypStage 0-I } & \multicolumn{2}{|c|}{ ypStage II-III } & \multirow[b]{2}{*}{ P-value } \\
\hline & $\mathrm{n}$ & $\%$ & $\mathrm{n}$ & $\%$ & \\
\hline \multicolumn{5}{|l|}{ Age (years) } & 0.896 \\
\hline$<65$ & 9 & 32.1 & 19 & 67.9 & \\
\hline$\geq 65$ & 7 & 30.4 & 16 & 69.6 & \\
\hline \multicolumn{5}{|l|}{ Gender } & 0.524 \\
\hline Male & 10 & 28.6 & 25 & 71.4 & \\
\hline Female & 6 & 37.5 & 10 & 62.5 & \\
\hline \multicolumn{5}{|l|}{ Tumor location } & 0.474 \\
\hline Low $^{b}$ & 2 & 20.0 & 8 & 80.0 & \\
\hline Middle-upper & 14 & 34.1 & 27 & 65.9 & \\
\hline \multicolumn{5}{|l|}{$\mathrm{cT}$} & 0.049 \\
\hline $\mathrm{T} 2$ & 3 & 60.0 & 2 & 40.0 & \\
\hline $\mathrm{T} 3$ & 11 & 34.4 & 21 & 65.6 & \\
\hline $\mathrm{T} 4$ & 2 & 14.3 & 12 & 85.7 & \\
\hline \multicolumn{5}{|l|}{$\mathrm{cN}$} & 0.054 \\
\hline $\mathrm{cN}-$ & 6 & 60.0 & 4 & 40.0 & \\
\hline $\mathrm{cN}+$ & 10 & 24.4 & 31 & 75.6 & \\
\hline \multicolumn{5}{|l|}{ Tumor size $(\mathrm{cm})$} & 0.003 \\
\hline$<5$ & 13 & 50.0 & 13 & 50.0 & \\
\hline$\geq 5$ & 3 & 12.0 & 22 & 88.0 & \\
\hline \multicolumn{5}{|l|}{ CEA (ng/ml) } & $<0.001$ \\
\hline$\leq 5$ & 13 & 54.2 & 11 & 45.8 & \\
\hline$>5$ & 1 & 4.8 & 20 & 95.2 & \\
\hline \multicolumn{5}{|l|}{ mSUV } & 0.155 \\
\hline$<10$ & 8 & 50.0 & 8 & 50.0 & \\
\hline$\geq 10$ & 8 & 28.6 & 20 & 71.4 & \\
\hline \multicolumn{5}{|c|}{ CRT-surgery interval (weeks) } & 0.466 \\
\hline$<7$ & 2 & 18.2 & 9 & 81.8 & \\
\hline$\geq 7$ & 14 & 35.0 & 26 & 65.0 & \\
\hline
\end{tabular}

${ }^{a}$ Chi-square or Fisher's exact test. ${ }^{\mathrm{b}} \leq 5 \mathrm{~cm}$ from the anal verge to the distal end of the tumor. CEA, carcinoembryonic antigen; mSUV, maximum standardized uptake value; CRT, chemoradiotherapy.

were independently associated with downstaging, another important criterion of response of LARC to CRT. The present study additionally demonstrated that CEA levels were associated with tumor size reduction.

The primary purpose of preoperative RT or CRT for rectal cancer is to decrease the risk of local disease recurrence (1). However, substantial progress in local recurrence rate reduction has been achieved using total mesorectal excision (13). An adequate oncological radical resection, with complete distal and radial clearance, and en bloc resection of the lympho-adipose tissue surrounding the rectum by total mesorectal excision, yields local recurrence rates of $4-10 \%$ after surgery alone (14). If the local recurrence rate after surgery alone is hypothesized to be $10 \%$, and if the addition of preoperative CRT cuts that down to half, then administering preoperative CRT to all LARC patients would have resulted in overtreatment in $\sim 95 \%$ of the patients. The introduction of
MRI to rectal cancer staging allows one to estimate the width of the circumferential resection margin, which is a strong predictor of the local recurrence risk (15). Selective use of preoperative treatments for rectal cancer has been suggested based on pretreatment MRI findings $(16,17)$. The need for preoperative CRT in LARC patients may be further assessed using pre-CRT CEA data. The present study demonstrated that pretreatment CEA levels are associated with downstaging, a valid surrogate marker of long-term disease recurrence (18).

A recent update report from a seminal randomized controlled trial that compared preoperative and postoperative CRT for LARC, indicated that the absolute difference in local recurrence rate decreased after a long (11-year) follow-up (19). The authors hypothesized that preoperative CRT only postpones local recurrence. Considering the minimal benefits conferred by local disease control, the major advantage of preoperative CRT may be the possible avoidance of major 
Table II. Univariate analysis of factors associated with downsizing of locally advanced rectal cancer.

\begin{tabular}{|c|c|c|c|c|c|}
\hline \multirow[b]{2}{*}{ Factors } & \multicolumn{2}{|c|}{ Downsizing $>60 \%$} & \multicolumn{2}{|c|}{ Downsizing $\leq 60 \%$} & \multirow[b]{2}{*}{ P-value ${ }^{a}$} \\
\hline & $\mathrm{n}$ & $\%$ & $\mathrm{n}$ & $\%$ & \\
\hline \multicolumn{5}{|l|}{ Age (years) } & 0.200 \\
\hline$<65$ & 16 & 57.1 & 12 & 42.9 & \\
\hline$\geq 65$ & 9 & 39.1 & 14 & 60.9 & \\
\hline \multicolumn{5}{|l|}{ Gender } & 0.193 \\
\hline Male & 15 & 42.9 & 20 & 57.1 & \\
\hline Female & 10 & 62.5 & 6 & 37.5 & \\
\hline \multicolumn{5}{|l|}{ Tumor location } & 0.499 \\
\hline Low $^{b}$ & 6 & 60.0 & 4 & 40.0 & \\
\hline Middle-upper & 19 & 46.3 & 22 & 53.7 & \\
\hline \multicolumn{5}{|l|}{$\mathrm{cT}$} & 0.252 \\
\hline $\mathrm{T} 2$ & 4 & 80.0 & 1 & 20.0 & \\
\hline T3 & 15 & 46.9 & 17 & 53.1 & \\
\hline $\mathrm{T} 4$ & 6 & 42.9 & 8 & 57.1 & \\
\hline \multicolumn{5}{|l|}{$\mathrm{cN}$} & 0.726 \\
\hline $\mathrm{cN}-$ & 4 & 40.0 & 6 & 60.0 & \\
\hline $\mathrm{cN}+$ & 21 & 51.2 & 20 & 48.8 & \\
\hline \multicolumn{5}{|l|}{ Tumor size $(\mathrm{cm})$} & 0.482 \\
\hline$<5$ & 14 & 53.8 & 12 & 46.2 & \\
\hline$\geq 5$ & 11 & 44.0 & 14 & 56.0 & \\
\hline \multicolumn{5}{|l|}{ CEA (ng/ml) } & 0.012 \\
\hline$\leq 5$ & 17 & 70.8 & 7 & 29.2 & \\
\hline$>5$ & 7 & 33.3 & 14 & 66.7 & \\
\hline \multicolumn{5}{|l|}{ mSUV } & 0.690 \\
\hline$<10$ & 9 & 56.3 & 7 & 43.8 & \\
\hline$\geq 10$ & 14 & 50.0 & 14 & 50.0 & \\
\hline \multicolumn{5}{|c|}{ CRT-surgery interval (weeks) } & 0.789 \\
\hline$<7$ & 5 & 45.5 & 6 & 54.5 & \\
\hline$\geq 7$ & 20 & 50.0 & 20 & 50.0 & \\
\hline
\end{tabular}

${ }^{a}$ Chi-square or Fisher's exact test. ${ }^{\mathrm{b}} \leq 5 \mathrm{~cm}$ from the anal verge to the distal end of the tumor. CEA, carcinoembryonic antigen; mSUV, maximum standardized uptake value; CRT, chemoradiotherapy.

surgical resection through CRT-induced tumor regression (3). For a subset of patients exhibiting a complete to near-complete response to preoperative CRT, experimental approaches, i.e., local excision or no surgery ('wait-and-see' policy) have been attempted $(6,20)$. While the necessity of preoperative CRT to improve long-term local disease control may be diminishing, the importance of preoperative CRT for possible conservative surgery (or even no surgery) continues to increase; accordingly, the value of pretreatment CEA levels for predicting CRT response will continue to increase.

This study was limited by its retrospective design and relatively small sample size. Smoking status may affect serum CEA levels, but the retrospective chart review method employed herein was unable to fully capture this information (10). Prospective studies including a larger patient sample are required to validate the current findings.
In conclusion, pretreatment serum CEA levels were found to be significantly associated with the downstaging and downsizing of LARC following preoperative CRT. This parameter represents a valuable tool that may facilitate treatment personalization for LARC patients.

\section{Acknowledgements}

The present study was supported by the Soonchunhyang University Research Fund.

\section{References}

1. Sauer R, Becker H, Hohenberger W, Rödel C, Wittekind C, Fietkau R, Martus P, Tschmelitsch J, Hager E, Hess CF, et al: Preoperative versus postoperative chemoradiotherapy for rectal cancer. N Engl J Med 351: 1731-1740, 2004. 
2. Lee JW, Lee JH, Kim JG, Oh ST, Chung HJ, Lee MA, Chun HG, Jeong SM, Yoon SC and Jang HS: Comparison between preoperative and postoperative concurrent chemoradiotherapy for rectal cancer: An institutional analysis. Radiat Oncol J 31 : $155-161,2013$.

3. Habr-Gama A, Gama-Rodrigues J and Perez RO: Is tailoring treatment of rectal cancer the only true benefit of long-course neoadjuvant chemoradiation? Dis Colon Rectum 56: 264-266, 2013.

4. Fleming FJ and Monson JR: The contemporary (over)treatment of rectal cancer: The goldilocks effect. Dis Colon Rectum 57: 403-406, 2014

5. Yeo SG, Kim DY, Kim TH, Chang HJ, Oh JH, Park W, Choi DH, Nam H, Kim JS, Cho MJ, et al: Pathologic complete response of primary tumor following preoperative chemoradiotherapy for locally advanced rectal cancer: Long-term outcomes and prognostic significance of pathologic nodal status (KROG 09-01). Ann Surg 252: 998-1004, 2010.

6. Yeo SG, Kim DY and Oh JH: Long-term survival without surgery following a complete response to pre-operative chemoradiotherapy for rectal cancer: A case series. Oncol Lett 6: 1573-1576, 2013.

7. Edge SB, Byrd DR, Compton CC, Fritz AG, Greene FL and Trotti A (eds): AJCC Cancer Staging Manual. 7th edition. Springer, New York, 2010.

8. Compton C, Fenoglio-Preiser CM, Pettigrew N and Fielding LP: American Joint Committee on Cancer prognostic factors consensus conference: Colorectal Working Group. Cancer 88 : 1739-1757, 2000

9. Park YA, Sohn SK, Seong J, Baik SH, Lee KY, Kim NK and Cho CW: Serum CEA as a predictor for the response to preoperative chemoradiation in rectal cancer. J Surg Oncol 93: 145-150, 2006.

10. Wallin U, Rothenberger D, Lowry A, Luepker R and Mellgren A: CEA - a predictor for pathologic complete response after neoadjuvant therapy for rectal cancer. Dis Colon Rectum 56: 859-868, 2013.

11. Restivo A, Zorcolo L, Cocco IM, Manunza R, Margiani C, Marongiu L and Casula G: Elevated CEA levels and low distance of the tumor from the anal verge are predictors of incomplete response to chemoradiation in patients with rectal cancer. Ann Surg Oncol 20: 864-871, 2013.
12. Yoon SM, Kim DY, Kim TH, Jung KH, Chang HJ, Koom WS, Lim SB, Choi HS, Jeong SY and Park JG: Clinical parameters predicting pathologic tumor response after preoperative chemoradiotherapy for rectal cancer. Int J Radiat Oncol Biol Phys 69: 1167-1172, 2007.

13. Sautter-Bihl ML, Hohenberger W, Fietkau R, Rödel C, Schmidberger H and Sauer R: Rectal cancer: When is the local recurrence risk low enough to refrain from the aim to prevent it? Strahlenther Onkol 189: 105-110, 2013.

14. Zoccali M and Fichera A: Role of radiation in intermediate-risk rectal cancer. Ann Surg Oncol 19: 126-130, 2012.

15. Taylor FG, Quirke P, Heald RJ, Moran B, Blomqvist L, Swift I, Sebag-Montefiore DJ, Tekkis P and Brown G; MERCURY study group: Preoperative high-resolution magnetic resonance imaging can identify good prognosis stage I, II, and III rectal cancer best managed by surgery alone: A prospective, multicenter, European study. Ann Surg 253: 711-719, 2011.

16. Frasson M, Garcia-Granero E, Roda D, Flor-Lorente B, Roselló S, Esclapez P, Faus C, Navarro S, Campos S and Cervantes A: Preoperative chemoradiation may not always be needed for patients with T3 and T2N+ rectal cancer. Cancer 117: 3118-3125, 2011.

17. Hawkes EA, Cunningham D, Tait D, Brown G and Chau I: Neoadjuvant chemotherapy alone for early-stage rectal cancer: An evolving paradigm? Semin Radiat Oncol 21: 196-202, 2011.

18. Yeo SG, Kim DY, Park JW, Choi HS, Oh JH, Kim SY, Chang HJ, Kim TH and Sohn DK: Stage-to-stage comparison of preoperative and postoperative chemoradiotherapy for T3 mid or distal rectal cancer. Int J Radiat Oncol Biol Phys 82: 856-862, 2012.

19. Sauer R, Liersch T, Merkel S, Fietkau R, Hohenberger W, Hess C, Becker H, Raab HR, Villanueva MT, Witzigmann H, et al: Preoperative versus postoperative chemoradiotherapy for locally advanced rectal cancer: Results of the German CAO/ARO/AIO-94 randomized phase III trial after a median follow-up of 11 years. J Clin Oncol 30: 1926-1933, 2012.

20. Lee NK, Kim DY, Kim SY, Oh JH, Park W, Choi DH, Nam TK and Lee KJ: Clinical outcomes of local excision following preoperative chemoradiotherapy for locally advanced rectal cancer. Cancer Res Treat 46: 158-164, 2014. 